\title{
The Minichromosome Maintenance Replicative Helicase
}

\author{
Stephen D. Bell ${ }^{1}$ and Michael R. Botchan ${ }^{2}$ \\ ${ }^{1}$ Molecular and Cellular Biochemistry Department, Biology Department, Indiana University, Bloomington, \\ Indiana 47405 \\ ${ }^{2}$ Department of Molecular and Cell Biology, Division of Biochemistry and Molecular Biology, \\ University of California, Berkeley, Berkeley, California 94720 \\ Correspondence: stedbell@indiana.edu; mbotchan@uclink.berkeley.edu
}

The eukaryotic replicative helicase, the minichromosome maintenance (MCM) complex, is composed of six distinct, but related, subunits $\mathrm{MCM}(2-7)$. The relationship between the sequences of the subunits indicates that they are derived from a common ancestor and indeed, present-day archaea possess a homohexameric MCM. Recent progress in the biochemical and structural studies of both eukaryal and archaeal MCM complexes are beginning to shed light on the mechanisms of action of this key component of the replisome.

$\mathrm{T}_{\mathrm{c}}^{\mathrm{h}}$ he minichromosome maintenance $(\mathrm{MCM})$ complex subunits are members of the $\mathrm{AAA}^{+}$ superfamily of ATPases and thus use energy derived from cycles of ATP binding and hydrolysis to move or reorganize bound substrates. In the case of the MCM complex, the energy is harnessed to effect DNA unwinding. The $\mathrm{AAA}^{+}$proteins can be classified into seven distinct clades, based on the topography of their active sites. MCMs are members of clade 7, being characterized by the presence of an additional $\alpha$-helix when compared with the classical $\mathrm{AAA}^{+}$fold (Iyer et al. 2004; Erzberger and Berger 2006). In addition to the $\mathrm{AAA}^{+}$domain, MCMs also have an amino-terminal domain (NTD) that plays a role in higher-order structure assembly. Finally, following the $\mathrm{AAA}^{+}$domain is a degenerate winged helix $(\mathrm{wH})$ structure (Fig. 1). Although the archaeal MCMs possess this simple NTD-AAA ${ }^{+}-\mathrm{wH}$ domain architecture, many of the eukaryal $\mathrm{MCM}(2-7)$ subunits are embellished with amino- or carboxy-terminal extensions that play roles in the regulation or recruitment of $\operatorname{MCM}(2-7)$. The eukaryal MCM complex is an important target for regulatory posttranslational modifications; the nature and consequences of these modifications are dealt with in Bell and Kaguni (2013), Tanaka and Araki (2013), and Siddiqui et al. (2013). Much of what we know regarding the inner workings of the MCM helicase has been learned from structural and mechanistic studies of the simple archaeal model (Sakakibara et al. 2009). Additionally, the crystal structures of distantly related superfamily three helicases, SV40 LTAg, and the E1 helicase of bovine papilloma virus, have provided important structural frameworks for understanding the mode of action of hexameric helicases (Gai et al. 2004; Enemark and Joshua-Tor 2006, 2008).

Editors: Stephen D. Bell, Marcel Méchali, and Melvin L. DePamphilis

Additional Perspectives on DNA Replication available at www.cshperspectives.org

Copyright (C) 2013 Cold Spring Harbor Laboratory Press; all rights reserved; doi: 10.1101/cshperspect.a012807

Cite this article as Cold Spring Harb Perspect Biol 2013;5:a012807 
S.D. Bell and M.R. Botchan

A
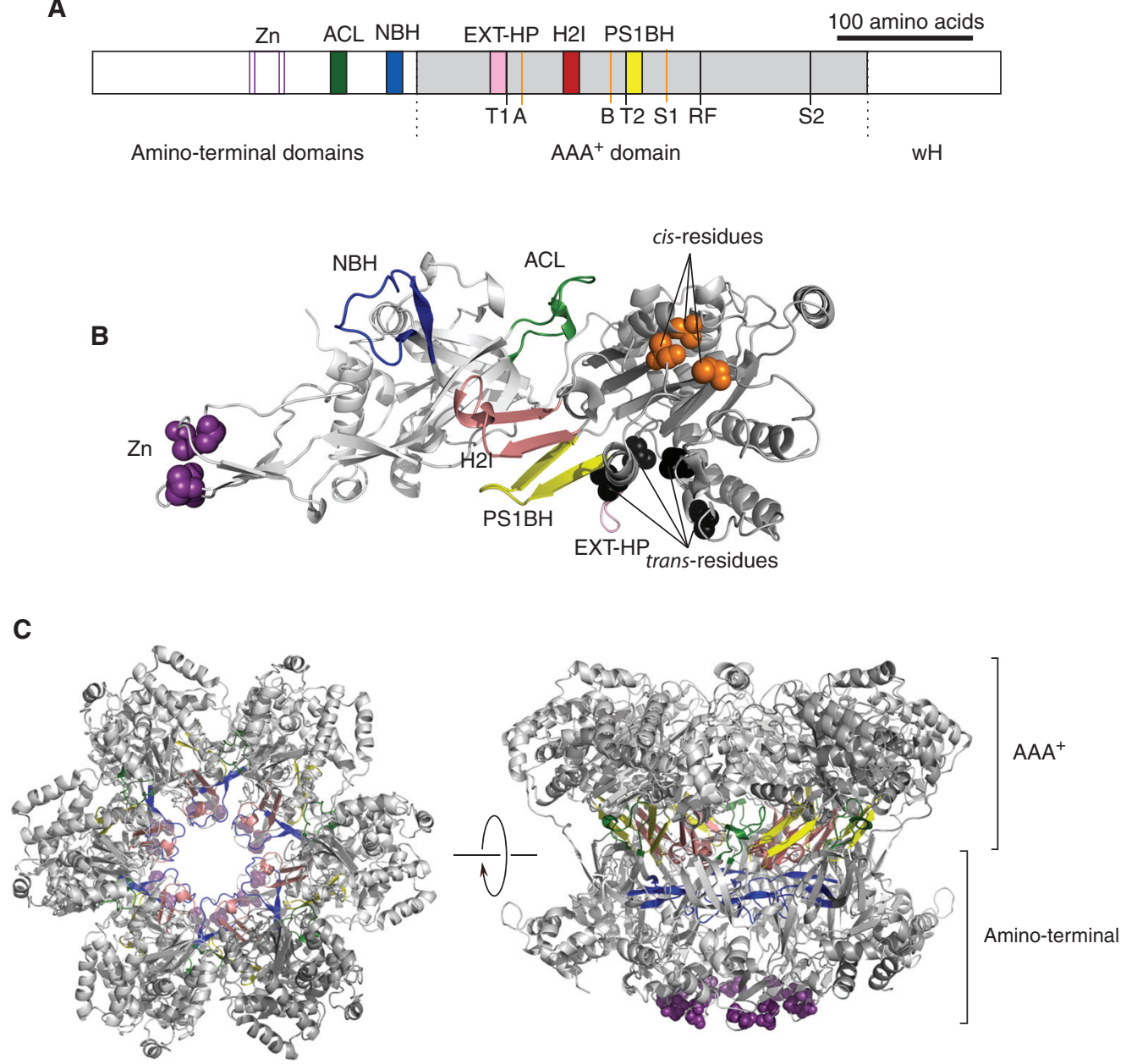

Figure 1. (A) Linear representation of a monomer of the archaeal MCM. (Gray) The central $\mathrm{AAA}^{+}$domain; (white) the flanking amino-terminal domains and winged helix $(\mathrm{wH})$. The position of key secondary structural elements-(Zn) zinc-binding; (ACL) allosteric communication loop; (NBH) amino-terminal $\beta$-hairpin; (EXT$\mathrm{HP}$ ) external $\beta$-hairpin; (H2I) helix 2 insert; and (PS1BH) pre-sensor $1 \beta$-hairpin-are indicated above the figure and shown by colored blocks, the colors corresponding to those used in panels $B$ and $C$. Key residues involved in the ATPase active site are indicated below the figure. (Orange lines) A, B, and S1, shown as orange lines are the Walker A lysine (K346), Walker B glutamate (E404), and Sensor 1 asparagine (N448), respectively, and constitute "cis"-acting residues. (Black lines) "Trans"-acting residues T1 (R331), T2 (Q423), arginine finger (R473), and Sensor 2 (R560). Numbering is from SsoMCM.(B) Structure of a monomer of SsoMCM (lacking detail of the $\mathrm{wH}$ domain). Secondary structure elements are labeled and colored in cartoon format, using the color scheme in panel A. (Purple spheres) The atoms of the zinc-coordinating residues; (orange spheres) the cisacting residues; (black spheres) the trans-residues.(C) Model of a symmetric hexamer of SsoMCM. (Left) View down the central cavity of SsoMCM, looking from the carboxy-terminal face. (Right) The same hexamer rotated $90^{\circ}$ to show a side view. The two tiers corresponding to the amino-terminal and $\mathrm{AAA}^{+}$domains are indicated. The color scheme is as in panels $A$ and $B$. Panels $B$ and $C$ were generated from PDB entry 3F9V using PyMOL (http://www.pymol.org). 
In the following, we shall describe structural and mechanistic insights derived from studies of the simpler archaeal MCMs before extending our discussion to the eukaryotic assembly.

\section{INSIGHTS FROM STRUCTURAL BIOLOGY}

Initial electron microscopy studies of Methanothermobacter thermautotrophicus (Mth) MCM revealed a double hexamer structure with the component hexamers joined in a head-to-head manner (Chong et al. 2000). Subsequent studies revealed a range of different forms of the same protein, including single hexamer and single heptamer rings and filaments (Yu et al. 2002; Pape et al. 2003; Chen et al. 2005). The ring structures all have a wide central pore, large enough to potentially accommodate single- or double-stranded DNA. An important step forward came with X-ray crystallographic studies that revealed the structure of residues 2-286 comprising the amino-terminal domains of the MthMCM (Fletcher et al. 2003). Strikingly, this formed a head-to-head double hexamer ring. The central channel, of minimal diameter of $23 \AA$, was ringed by $\beta$-hairpin elements, one contributed by each protomer. The channel is highly positively charged, and conserved basic residues are located at the tips of the $\beta$-hairpins. DNA-binding studies revealed that MthMCM (2-286) was able to bind to both dsDNA and ssDNA in a manner dependent on the basic residues at the tips of the hairpins (Fletcher et al. 2003).

In addition to the $\beta$-hairpin structures, the amino-terminal domains of MCM possess a zinc-binding motif. Biochemical studies with MthMCM have implicated this motif in stabilizing the amino-terminal domains and thus facilitating interhexamer interactions (Poplawski et al. 2001; Fletcher et al. 2003, 2005; Kasiviswanathan et al. 2004). However, the physiological relevance of the double-hexamer form of MthMCM has been drawn into question by the observation that other all other archaeal MCMs characterized to date exist as single hexamers. A study by Kelman and colleagues has helped resolve this issue (Shin et al. 2009). Using gel fil- tration approaches, it was revealed that low temperature helped stabilize the double hexamer, although higher temperatures favored a single hexameric form. The experiments were somewhat constrained by the inability of conventional chromatography equipment to operate at temperatures above $50^{\circ} \mathrm{C}$, and it was therefore not possible to perform the experiment at $60^{\circ} \mathrm{C}$, the normal growth temperature of Mth! Nevertheless, these experiments, in conjunction with studies of multimer formation under physiological salt concentrations ( $\sim 800 \mathrm{~mm}$ potassium) and helicase activity assays, provided strong support for the active form of MthMCM, as found for other archaeal MCMs, to be a single hexamer (Shin et al. 2009).

To date, there are no high-resolution structures available for MCM hexamers from any archaea; however, EM reconstructions of hexameric rings of MthMCM have been generated, revealing a two-tiered structure (Costa and Onesti 2009). Modeling of the amino-terminal domains into the EM envelope has indicated that one tier is composed of the amino-terminal domains, and the other tier by the $\mathrm{AAA}^{+}$domain. More recently, crystal structures of a monomer of an inactive MCM from Methanopyrus kandleri and of a monomer of Sulfolobus solfataricus MCM residues 7-601 (lacking detail of the $85 \mathrm{wH}$ domain) have been determined to $1.9 \AA$ and $4.35 \AA$, respectively (Brewster et al. 2008; Bae et al. 2009), allowing the generation of a series of first-generation models for the hexameric assembly (Fig. 1B,C). These models use a sixfold symmetric rotation of the monomer around an axis, and from what is understood from other $\mathrm{AAA}^{+}$assemblies, it is likely that important asymmetry with regard to mechanism will be uncovered with high-resolution structures of the hexamer (Martin et al. 2005).

\section{HAIRPINS IN MCM}

In common with the distantly related superfamily three (SF3) helicases encoded by several viruses (Enemark and Joshua-Tor 2008), the $\mathrm{AAA}^{+}$fold of MCM is embellished by DNAinteracting $\beta$-hairpin motifs. However, in contrast to the single $\beta$-hairpin in the SF3 helicases, 
three such elements have been described in the $\mathrm{MCM} \mathrm{AAA}^{+}$core. Starting from the carboxyterminal end and proceeding toward the amino terminus of the $\mathrm{AAA}^{+}$domain of the $\mathrm{Mcms}$, the first hairpin encountered is the presensor 1 $\beta$-hairpin (PS1BH). This hairpin, with homology to the hairpin of the SF3 family members, extends into the central pore of the SF3 enzymes and plays a pivotal role in the helicase activity (McGeoch et al. 2005). Substitution by alanine of a conserved basic residue found near the tip of the $\beta$-hairpin only modestly impaired DNA binding by SsoMCM. Notably, however, the helicase activity of the MCM was abrogated by the mutation. The analogous $\beta$-hairpin in SF3 helicases has been shown to undergo significant movement (up to $17 \AA$ in the case of SV40 LTAg) during the ATP utilization cycle of the helicase (Gai et al. 2004). Furthermore, the PS1BH elements in the crystal structure of the BPV E1 helicase bound to DNA stack in a staircase, the position of a given $\beta$-hairpin being dictated by the nucleotide status of the relevant subunit (Enemark and Joshua-Tor 2006). As a subunit goes through its ATP binding, hydrolysis, and release cycle, the $\beta$-hairpin repositions, thereby driving the helicase along DNA. The conservation of the hairpin in MCM and its essential nature for the helicase activity of the enzyme strongly hint at a similar mode of action for this element. Indeed, evidence has been found for a relative repositioning of the PS1BH in a nucleotide-dependent manner (Barry et al. 2009). Interestingly, the second $\beta$-hairpin element in MCM, the H2I (helix 2 insert) has also been shown to undergo nucleotide-dependent repositioning (Jenkinson and Chong 2006). Studies in MthMCM revealed changes in fluorescence of the single tryptophan residue in this protein, fortuitously located in the H2I, in the presence of nucleotide. Modulation of fluorescence was also observed in the presence of DNA, leading to the proposal that the H2I could project into the central cavity of the MCM hexamer (Jenkinson and Chong 2006). This proposal has been substantiated and extended by subsequent structural work (Brewster et al. 2008). Indeed, the crystal structure of SsoMCM reveals very close apposition of H2I and PS1BH, suggesting that these two elements may function as a single unit (Beattie and Bell 2011).

The third $\beta$-hairpin element, the EXT-hairpin, in MCM's $\mathrm{AAA}^{+}$domain lies on the outer surface of the hexamer. It is relatively poorly conserved but may play a further role in DNA binding, as discussed below.

MCM is a $3^{\prime}$-to- $5^{\prime}$ helicase; thus, it translocates along DNA in a $3^{\prime}$-to- $5^{\prime}$ direction, and this leads to DNA melting. Several models have been proposed for how the helicase activity is effected. Several fluorescence resonance energy transfer (FRET) studies have provided support for a simple steric exclusion model in which a hexamer of MCM translocates along single-stranded DNA, displacing a second strand of DNA ahead of it (Fig. 2) (McGeoch et al. 2005; Rothenberg et al. 2007; Graham et al. 2011). Initial ensemble FRET studies indicated that MCM could slide onto the $3^{\prime}$-protruding strand of

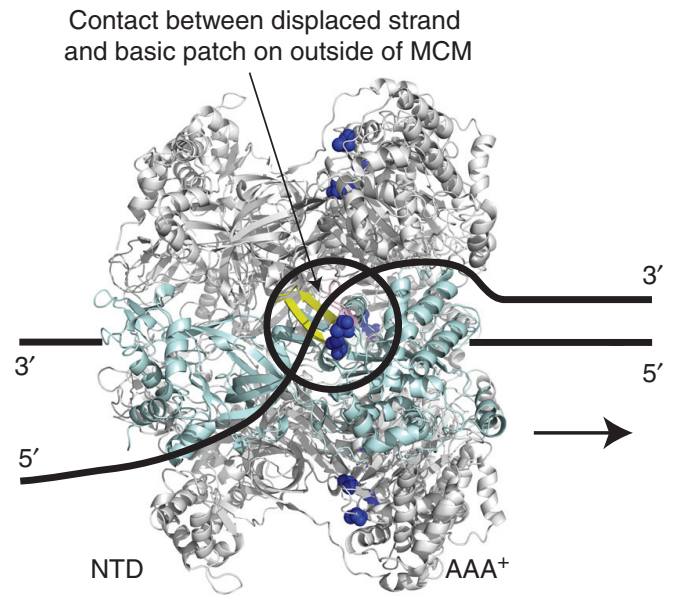

Figure 2. Model for MCM's helicase activity. MCM is shown to $3^{\prime}$-to- $5^{\prime}$ translocate along a single strand of DNA that passes through the central cavity of the hexamer, one subunit of which is shown in pale blue. The displaced strand makes transient contacts with the outer surface of the hexamer (Rothenberg et al. 2007; Graham et al. 2011). Residues K323 and R440 of SsoMCM have been implicated in this contact (dark blue spheres) (Graham et al. 2012). (Yellow ribbon) The adjacent PS1BH. Aside from the potential role of these charged residues in contacting the displaced strand, nothing is known regarding the details of the trajectory of the displaced strand on the outer surface of the helicase. 
a Y-shaped DNA substrate with the $\mathrm{AAA}^{+}$domains of the protein facing the duplex junction and the amino-terminal domains trailing behind (McGeoch et al. 2005). More recent single-molecule FRET experiments have revealed that MCM interacts more stably with a fork substrate than with a simple $3^{\prime}$-extended molecule - suggesting that the displaced strand might somehow stabilize the assembly and perhaps bind this strand on surfaces topologically external to the central channel (Rothenberg et al. 2007). Significantly, single-molecule FRET studies examining interactions between the $3^{\prime}$ - and $5^{\prime}$-extended arms of the substrate revealed that the displaced strand did, indeed, undergo a dynamic interaction with MCM (Rothenberg et al. 2007). Subsequent work has confirmed the initial observations and provided evidence that charged residues on the outer surface of MCM (K323, located at the base of the EXT-hairpin, and R440, near the base of the PS1BH, of SsoMCM) (Fig. 2), were important for this interaction with the displaced strand (Graham et al. 2011). These transient contacts between the displaced strand and the outer surface of MCM could have several consequences - first, they might stabilize the hexameric assembly on DNA; second, they could facilitate processive DNA unwinding by the helicase; and third, they may help channel the displaced strand, the lagging strand template, to other components of the replisome (see below).

\section{INTERSUBUNIT COMMUNICATION}

Although the $\mathrm{AAA}^{+}$domains of MCM are the motors that drive the helicase along its substrate, the amino-terminal domains also play pivotal roles in governing helicase action. Deletion studies have revealed that the amino-terminal domains are not required for helicase activity in vitro, but their absence alters the range of DNA substrates that the MCM can melt (Barry et al. 2007). The presence of the amino-terminal domains also enhances the processivity of the helicase in a manner dependent on the ability of the amino-terminal domains to interact with DNA via the amino-terminal $\beta$-hairpin (Barry et al. 2007). The contacts between this fourth
MCM $\beta$-hairpin and DNA are distal from the $\mathrm{AAA}^{+}$core where ATP binding and hydrolysis cycles drive the conformational changes in the motor that, in turn, exert the forces required for the power stroke; nevertheless, such contacts are critical for substrate affinity. It thus seems likely that the amino-terminal domains are not acting simply as a passive sliding clamp trailing behind the $\mathrm{AAA}^{+}$domains but rather play a more active role coordinating interprotomer interactions within the MCM hexamer.

Like many $\mathrm{AAA}^{+}$proteins, the ATP-binding sites in MCM are located at the interface between protomers (Iyer et al. 2004; Erzberger and Berger 2006). Residues from both flanking subunits coordinate ATP, the classical examples being the residues constituting the Walker $\mathrm{A}$, Walker B, and Sensor 1 motif of a given active site (termed cis-residues in the following) and elements from the flanking protomer that contribute trans-acting residues, the classic example being the arginine finger. The intersubunit nature of the active sites in conjunction with the ring-shaped assembly opens the door to cooperative interactions between active sites around the MCM ring. Indeed, several other ring-shaped helicases and translocases show high cooperativity between active sites, such that incorporation of a single mutant site into an otherwise wildtype ring results in complete abrogation of enzyme activity (Crampton et al. 2006). However, not all ring-shaped $\mathrm{AAA}^{+}$machines show such high cooperativity. For example, studies of the protein unfoldase ClpX revealed that ATP hydrolysis by a given active site was essentially probabilistic in nature and that there was no strict requirement for cooperative or sequential hydrolysis events around the ring (Martin et al. 2005). Mutant doping experiments with SsoMCM revealed an intriguing intermediate behavior, with localized cooperativity between adjacent pairs of active sites in SsoMCM (Moreau et al. 2007). More specifically, mutation of an individual active site also impaired function at the neighboring site, but the toxic effect spread no further around the ring. These results reveal that despite the homohexameric nature of the archaeal MCM, asymmetries exist at the functional level. 
Intriguingly, deletion of the amino-terminal domains abolished the cooperativity between $\mathrm{AAA}^{+}$domains (Barry et al. 2009). Mutational studies have revealed a role for a conserved loop, termed the allosteric communication loop (ACL), at the $\mathrm{AAA}^{+}$-proximal face of the amino-terminal domains in facilitating communication between protomers (Sakakibara et al. 2008; Barry et al. 2009). This loop was found to lie in close proximity to the PS1BH of the neighboring protomer. Furthermore, nucleotide-modulated contacts between the PS1BH and the ACL may, in turn, reposition the amino-terminal $\beta$-hairpin of the ACLcontaining subunit (Barry et al. 2009). The precise details of the mechanochemistry of the archaeal MCM awaits further structural elucidation; however, mutagenesis studies have implicated a series of conserved charged and polar residues in transducing information between the active site of the helicase and the PS1BH/ H2I assembly (Liew and Bell 2011). It is eagerly anticipated that higher-resolution structures of active forms of archaeal MCMs will be forthcoming in the near future.

Of course, the archaeal MCM does not function in isolation in unwinding DNA at the replication fork; rather, it will be integrated into a higher-order replisome assembly. Details of the organization of the archaeal replisome remain sketchy thus far, but several laboratories have characterized archaeal counterparts of the eukaryotic GINS complex as interacting with MCM (Marinsek et al. 2006; Labib and Gambus 2007; Yoshimochi et al. 2008; Ishino et al. 2011). Initially, archaeal GINS homologs were identified by bioinformatics approaches (Makarova et al. 2005). A subsequent yeast two-hybrid screen identified Gins23 (homologous to eukaryotic GINS components Psf2 and Psf3) as an interaction partner for the amino-terminal domains of SsoMCM (Marinsek et al. 2006). Purification of the endogenous Gins23 revealed association with a second GINS component homolog, Gins15 (homologous to eukaryotic Psf1 and Sld5). Gins23:Gins15 formed a 2:2 tetrameric complex. The Sulfolobus GINS complex copurified with a further protein, related to the ssDNA binding domain of RecJ (Marinsek et al. 2006). Importantly, recent bioinformatics studies have revealed a clear relationship between RecJ and the eukaryotic replication factor Cdc45 (Sanchez-Pulido and Ponting 2011). This association of a Cdc45 homolog with archaeal GINS is conserved across diverse archaea, because recent work has identified an RecJ/ Cdc45 homolog, GAN (GINS-associated nuclease), as a partner of the Thermococcus GINS complex (Li et al. 2010, 2011). Despite the association of the GINS/Cdc45 with SsoMCM, no effect on the helicase or ATPase activities of the MCM could be observed in vitro on mixing of the two complexes (Marinsek et al. 2006). In contrast, studies in Pyrococcus and Thermococcus have indicated a stimulation of the basal helicase activity of these species' MCM by their corresponding GINS complex (Yoshimochi et al. 2008; Ishino et al. 2011).

\section{THE EUKARYOTIC MCM COMPLEX}

Yeast plasmids that harbor only single origins of replication are sensitized for loss in cells with hypomorphic mutations in DNA replication genes. Tye first used this insight to identify genes required for "minichromosome maintenance." Therefore, genes encoding MCMs of widely different functions were initially identified through screens for plasmid maintenance, chromosome segregation, and cell cycle progression. The six genes called MCM 2-7 encode subunits of the eukaryal MCM complex and are now known to be the core of the eukaryotic replicative helicase. For excellent reviews of the identification of $\operatorname{MCM}(2-7)$ and evidence for their role as the replicative helicase, see Kearsey and Labib (1998) and Bochman and Schwacha (2009).

The six subunits of MCM assemble in a ring with a defined geometry of MCM5-3-7-4-6-2 (Fig. 3) (Crevel et al. 2001; Schwacha and Bell 2001; Davey et al. 2003). Although it has been known for a number of years that the subassembly of $\operatorname{MCM}(4,6,7)$ possesses robust helicase activity in vitro (Ishimi 1997) and despite the convincing evidence that $\operatorname{MCM}(2-7)$ was required for initiation and elongation of DNA replication in vivo (Labib et al. 2000), 

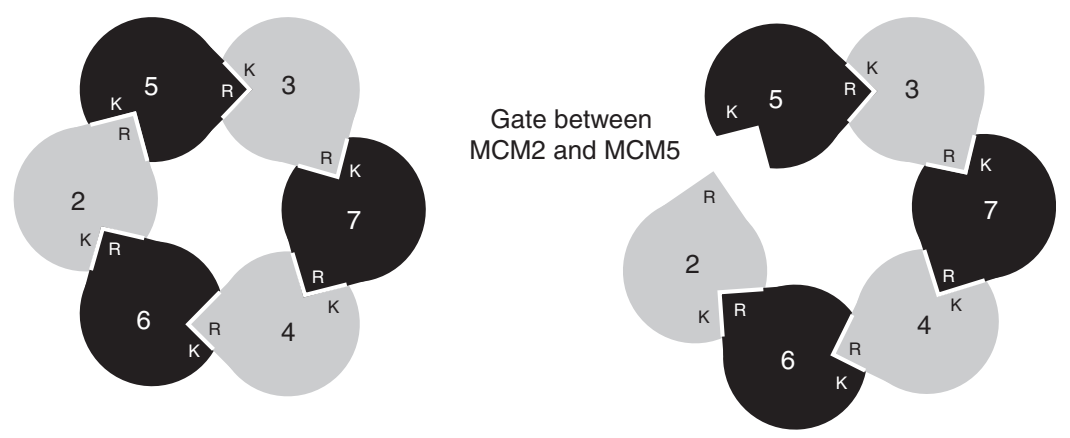

Figure 3. Organization of eukaryotic $\mathrm{MCM}(2-7)$. Interactions between protomers of MCM result in a heterohexamer of defined geometry (Ishimi 1997; Schwacha and Bell 2001; Davey et al. 2003). In the cartoon, the Ks indicate the cis-acting Walker A lysine residue of a given subunit, and the Rs show the trans-acting arginine fingers. Thus, the active site between protomers is contributed to by the cis surface of one protomer and the trans surface of its neighbor. As detailed in the text, considerable biochemical and structural data support the existence of a gate between subunits $\mathrm{Mcm} 2$ and $\mathrm{Mcm} 5$ (right panel).

in vitro evidence directly showing helicase activity of $\operatorname{MCM}(2-7)$ was lacking. However, two seminal advances have unambiguously shown that $\operatorname{MCM}(2-7)$ is a functional helicase. First, $\operatorname{MCM}(2-7)$ was shown to be the core of a larger complex with GINS and Cdc45, termed CMG. CMG possessed robust helicase activity in vitro (Moyer et al. 2006; Ilves et al. 2010). Second, studies of budding yeast $\operatorname{MCM}(2-7)$ revealed that the assembly had helicase activity but that this was dependent on specific buffer conditions, not previously tested (Bochman and Schwacha 2008). More specifically, the helicase activity is highly dependent on the choice of anion in the buffer, with activity being stimulated by glutamate and to a lesser extent acetate. There appears to be a degree of species specificity to these results, because studies with Drosophila $\operatorname{MCM}(2-7)$, purified as recombinant proteins from Sf9 cells, revealed this complex to have minimal helicase activity in glutamate buffer, contrasting with the robust activity of the analogous CMG complex (Ilves et al. 2010). The elevated helicase activity of CMG is probably in part due to the $\sim 300$ fold elevation of the $V_{\max }$ for ATP hydrolysis conferred on Drosophila MCM(2-7) by the association with GINS and Cdc45. Perhaps the association of the budding yeast GINS and Cdc45 proteins with the core $\mathrm{MCM}(2-7)$ proteins will also increase the specific activity of the yeast complex. Hence, the apparent differences may simply reflect different basal activities.

\section{A GATE IN MCM(2-7)}

Studies with purified Saccharomyces cerevisiae $\operatorname{MCM}(2-7)$ revealed that pre-incubation of the complex with ATP or a poorly hydrolyzable analog resulted in an impairment of the helicase to bind to covalently closed circular singlestranded DNA but had negligible effects on the ability of the complex to bind linear DNA (Bochman and Schwacha 2008). This result suggested that a gate may exist in MCM that is closed in the presence of ATP, thereby topologically excluding DNA from the central pore of the toroid. As described above, the ATPase sites of MCM lie between subunits, and thus a series of mutagenesis studies was performed to target individual interfaces systematically, rendering them incapable of binding ATP. Strikingly, mutation of either the Walker A lysine residue of MCM5 or the arginine finger of MCM2 rendered the $\operatorname{MCM}(2-7)$ insensitive to ATP when binding circular single-stranded DNA. Thus, it appears that the interface between MCM2 and MCM 5 serves as an ATP-modulated gate through which ssDNA can pass (Bochman and Schwacha 2008). These conclusions, drawn from biochemical experiments, have received structural confirmation with the elucidation of EM reconstructions 
of purified recombinant Drosophila $\mathrm{MCM}(2-$ 7). Like the archaeal MCM, a two-tier structure was observed, corresponding to amino-terminal domains and $\mathrm{AAA}^{+}$domains (Costa et al. 2011). However, two distinct conformations of $\operatorname{MCM}(2-7)$ were observed. In one form, a near symmetric ring structure was seen that nevertheless possessed a notch in the $\mathrm{AAA}^{+}$tiers. The second, and more abundant, form had an asymmetric gapped or a lock washer configuration with an opening extending the full length of the MCM. In these conformations, the individual subunits were nonplanar and assumed a slight helical twist. Experiments using $\operatorname{MCM}(2-7)$ with individually tagged subunits revealed that the gap lay between MCM2 and MCM5 (Costa et al. 2011). Further work illustrated the structure of the Drosophila CMG complex. This revealed a more symmetrical ring-shaped configuration of the MCMs where closer interaction between surfaces for each of the subunits was detected, embellished by a handle of additional density on one side of the ring (Costa et al. 2011). More detailed analyses indicated that the MCM adopted the notched ring conformation seen with the isolated $\mathrm{MCM}(2-7)$. Incubation with $\mathrm{ADP} \cdot \mathrm{BeF}_{3}$ resulted in narrowing of the notch. Furthermore, the handle-like protuberance, corresponding to GINS/CDC45, wrapped around several subunits of MCM, including those, MCM2 and 5, contributing to the gap (Fig. 4). These observa-

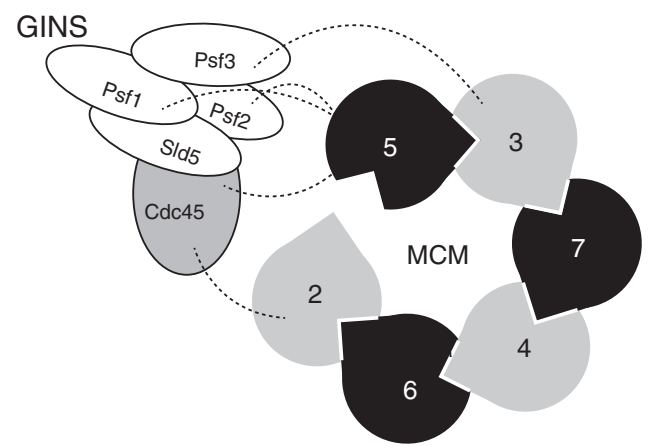

Figure 4. Interaction of the GINS components and Cdc45 with MCM. The cartoon indicates the open form of $\operatorname{MCM}(2-7)$ with dotted lines revealing the interactions between MCM subunits and Psf1, 2, 3, and Cdc45. tions led to the conclusion that the enhanced ATPase activity and helicase function of the CMG relative to the $\operatorname{MCM}(2-7)$ complex was likely affected allosterically by the closing of the MCM2 and 5 gate. This induced conformational change would enable the appropriate juxtaposition of the Walker A and Arg-finger surfaces of the different subunits to form an active site. It is tempting to speculate that the yeast MCM complex may also assume open and closed complex configurations (Remus et al. 2009). Furthermore, it is possible that, in the absence of yeast GINS and CDC45, an equilibrium with more of the complex in the closed form may exist than that observed with the Drosophila complex.

Taken together, these EM snapshots suggest a tantalizing model for how MCM might become activated at replication origins (Costa et al. 2011). At eukaryotic origins of replication, the MCM complex is loaded before its association with GINS and CDC45. To date, all of the available evidence indicates that MCM is loaded onto double-stranded DNA at origins (Remus et al. 2009). Thus, the open lock washer configuration observed for $\operatorname{MCM}(2-7)$ may correspond to a loading-proficient form of the helicase. Once loaded, MCM may isomerize to a planar form, possibly concomitant with localized DNA melting. The leading-strand template will be of the correct polarity to be stably bound by the $\beta$-hairpins in the heart of the MCM's pore, whereas the lagging-strand template could be displaced out via the gate. The extruded single-stranded DNA would then be trapped by the flanking GINS/CDC45 handle, recruited to the outer surface of the core helicase (Fig. 5). In this regard, it is enticing to note that the archaeal MCM has been shown to have DNA-binding surfaces on the outside of the helicase (see Fig. 2). Perhaps these could play a role in channeling the extruded DNA to the GINS/Cdc45 addendum on the core helicase (Rothenberg et al. 2007; Graham et al. 2011). The actual path of the "lagging" 5 ' strand in or around the external surface of the helicase remains an important area for investigation.

Thus, the CMG complex may have two pores, one passing through the center of the toroid of MCM by which the helicase motor 


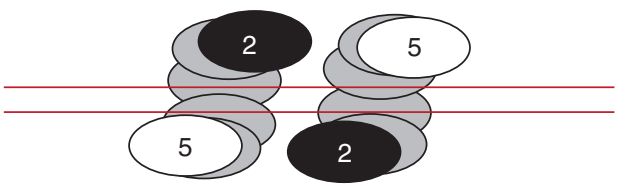

Reform planar MCM ring $\downarrow$ DNA melting and extrusion
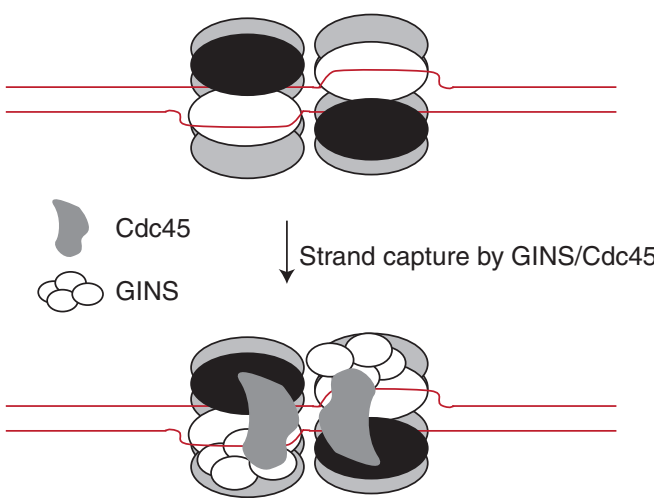

Figure 5. A speculative model for MCM loading and DNA melting at replication origins in eukarya and, by inference, archaea. Two open-gate hexamers of MCM are loaded at an origin. Ring closure occurs, concomitant with localized melting of DNA. One strand, the leading-strand template, is retained in the heart of the helicase; the other, the extruded lagging-strand template, is captured by the GINS/Cdc45 on the outer surface of the helicase assembly.

engages with the leading-strand template; and the other between the outer surface of MCM and GINS/CDC45 entrapping the laggingstrand template. It is possible that this additional binding may contribute significantly to the processivity of the helicase. We also note that such a model could explain why CMG has been observed to bind fork DNA substrates preferentially over $3^{\prime}$-extended molecules and with a 10 fold higher affinity than the isolated $\operatorname{MCM}(2-$ 7). Unpublished studies with the Drosophila CMG complex substantiate the anticipated parallels with the archaeal MCM complex. Mutations in the presensor-1 $\beta$-hairpins affect the affinity for substrate binding and collectively are required for helicase function. Furthermore, DNA/protein cross-linking experiments reveal a distinct pattern of MCM:DNA interactions with the leading $3^{\prime}$ strand when DNA probes are engineered with UV-sensitive $\mathrm{T}$ residues within the single-strand regions of a fork. However, no cross-linking is detected with protein when such residues are placed within the duplex stretch proximal to the nexus of the fork ( $\mathrm{JPe}$ savento, T Petojevic, and MR Botchan, unpubl.). These results indicate that the CMG translocates on the $3^{\prime}$ strand through tight contacts within the interior of the toroid, without duplex in intimate association with the protein or entering the channel.

Recent work using a Xenopus cell-free system has provided strong evidence that the eukaryotic MCM helicase preferentially translocates along a single strand of DNA (Fu et al. 2011). It was observed that the CMG complex could be stalled very effectively by a roadblock in the form of an intrastrand cross-link, a strand-specific biotin-streptavidin moiety, or a $20-\mathrm{nm}$ quantum dot attached to the leadingstrand template. In contrast, such roadblocks had much less effect when placed on the lagging-strand template. However, the laggingstrand blocks did still cause transient stalling, albeit to a lower level than seen with the leading strand. Furthermore, because the experiments were performed with cell extracts, it is conceivable that additional factors present in the extract facilitate bypass of a lagging-strand block. The lack of a strong effect of the lagging-strand template blocks indicates that the contacts that may occur with the secondary channel or the "outside surfaces" of the MCM ring are as not sterically constrained and are of lower affinity than those contacts made interior to the main channel. Understanding how other components such as the DNA polymerases, RPA, RFC, and the clamp loader are organized in contact with the CMG and perhaps affect the rates of unwinding will be challenges for the future.

\section{ACKNOWLEDGMENTS}

Work in S.D.B.'s laboratory is funded by the Wellcome Trust and the Biotechnology and Biological Sciences Research Councel (UK). Work in the Botchan laboratory is funded by NIH CA R37-30490. We apologize to those of our colleagues whose work we have not had space to cite. 
S.D. Bell and M.R. Botchan

\section{REFERENCES}

* Reference is also in this collection.

Bae B, Chen Y-H, Costa A, Onesti S, Brunzelle JS, Lin Y, Cann IKO, Nair SK. 2009. Insights into the architecture of the replicative helicase from the structure of an Archaeal MCM homolog. Structure 17: 211-222.

Barry ER, McGeoch AT, Kelman Z, Bell SD. 2007. Archaeal MCM has separable processivity, substrate choice and helicase domains. Nucleic Acids Res 35: 988-998.

Barry ER, Lovett JE, Costa A, Lea SM, Bell SD. 2009. Intersubunit allosteric communication mediated by a conserved loop in the MCM helicase. Proc Natl Acad Sci 106: $1051-1056$.

Beattie TR, Bell SD. 2011. Molecular machines in Archaeal DNA replication. Curr Opin Chem Biol 15: 614-619.

* Bell SP, Kaguni JM. 2013. Helicase loading at chromosomal origins of replication. Cold Spring Harb Perspect Biol doi: 10.1101/cshperspect.a010124.

Bochman ML, Schwacha A. 2008. The Mcm2-7 complex has in vitro helicase activity. Mol Cell 31: 287-293.

Bochman ML, Schwacha A. 2009. The Mcm complex: Unwinding the mechanism of a replicative helicase. Microbiol Mol Biol Rev 73: 652-683.

Brewster AS, Wang G, Yu X, Greenleaf WB, Maria Carazo J, Tjajadia M, Klein MG, Chen XS. 2008. Crystal structure of a near-full-length archaeal MCM: Functional insights for an AAA plus hexameric helicase. Proc Natl Acad Sci 105: 20191-20196.

Chen YJ, Yu XO, Kasiviswanathan R, Shin JH, Kelman Z, Egelman EH. 2005. Structural polymorphism of Methanothermobacter thermautotrophicus MCM. J Mol Biol 346: $389-394$

Chong JPJ, Hayashi MK, Simon MN, Xu RM, Stillman B. 2000. A double-hexamer archaeal minichromosome maintenance protein is an ATP-dependent DNA helicase. Proc Natl Acad Sci 97: 1530-1535.

Costa A, Onesti S. 2009. Structural biology of MCM helicases. Crit Rev Biochem Mol Biol 44: 326-342.

Costa A, Ilves I, Tamberg N, Petojevic T, Nogales E, Botchan MR, Berger JM. 2011. The structural basis for MCM2-7 helicase activation by GINS and Cdc45. Nat Struct Mol Biol 18: 471-477.

Crampton DJ, Mukherjee S, Richardson CC. 2006. DNAinduced switch from independent to sequential dTTP hydrolysis in the bacteriophage T7 DNA helicase. Mol Cell 21: $165-174$.

Crevel G, Ivetic A, Ohno K, Yamaguchi M, Cotterill S. 2001. Nearest neighbour analysis of MCM protein complexes in Drosophila melanogaster. Nucleic Acids Res 29: 48344842.

Davey MJ, Indiani C, O’Donnell M. 2003. Reconstitution of the $\mathrm{Mcm} 2-7 \mathrm{p}$ heterohexamer, subunit arrangement, and ATP site architecture. J Biol Chem 278: 4491-4499.

Enemark EJ, Joshua-Tor L. 2006. Mechanism of DNA translocation in a replicative hexameric helicase. Nature 442: $270-275$.

Enemark EJ, Joshua-Tor L. 2008. On helicases and other motor proteins. Curr Opin Struct Biol 18: 243-257.
Erzberger JP, Berger JM. 2006. Evolutionary relationships and structural mechanisms of AAA plus proteins. Annu Rev Biophys Biomol Struct 35: 93-114.

Fletcher RJ, Bishop BE, Leon RP, Sclafani RA, Ogata CM, Chen XJS. 2003. The structure and function of MCM from archaeal M. thermoautotrophicum. Nat Struct Biol 10: $160-167$.

Fletcher RJ, Shen JP, Gomez-Llorente Y, San Martin C, Carazo JM, Chen XJS. 2005. Double hexamer disruption and biochemical activities of Methanobacterium thermoautotrophicum MCM. J Biol Chem 280: 42405-42410.

Fu YV, Yardimci H, Long DT, Ho TV, Guainazzi A, Bermudez VP, Hurwitz J, van Oijen A, Schaerer OD, Walter JC. 2011. Selective bypass of a lagging strand roadblock by the eukaryotic replicative DNA helicase. Cell 146: $930-940$.

Gai DH, Zhao R, Li DW, Finkielstein CV, Chen XS. 2004. Mechanisms of conformational change for a replicative hexameric helicase of SV40 large tumor antigen. Cell 119: 47-60.

Graham BW, Schauer GD, Leuba SH, Trakselis MA. 2011. Steric exclusion and wrapping of the excluded DNA strand occurs along discrete external binding paths during MCM helicase unwinding. Nucleic Acids Res 39: 6585-6595.

Ilves I, Petojevic T, Pesavento JJ, Botchan MR. 2010. Activation of the MCM2-7 helicase by association with Cdc45 and GINS proteins. Mol Cell 37: 247-258.

Ishimi Y. 1997. A DNA helicase activity is associated with an MCM4, -6, and -7 protein complex. J Biol Chem 272: $24508-24513$.

Ishino S, Fujino S, Tomita H, Ogino H, Takao K, Daiyasu H, Kanai T, Atomi H, Ishino Y. 2011. Biochemical and genetical analyses of the three $m \mathrm{~cm}$ genes from the hyperthermophilic archaeon, Thermococcus kodakarensis. Genes Cells 16: 1176-1189.

Iyer LM, Leipe DD, Koonin EV, Aravind L. 2004. Evolutionary history and higher order classification of AAA plus ATPases. J Struct Biol 146: 11-31.

Jenkinson ER, Chong JPJ. 2006. Minichromosome maintenance helicase activity is controlled by $\mathrm{N}$ - and C-terminal motifs and requires the ATPase domain helix-2 insert. Proc Natl Acad Sci 103: 7613-7618.

Kasiviswanathan R, Shin JH, Melamud E, Kelman Z. 2004. Biochemical characterization of the Methanothermo bacter thermautotrophicus minichromosome maintenance (MCM) helicase N-terminal domains. J Biol Chem 279: 28358-28366.

Kearsey SE, Labib K. 1998. MCM proteins: Evolution, properties, and role in DNA replication. Biochim Biophys Acta 1398: $113-136$.

Labib K, Gambus A. 2007. A key role for the GINS complex at DNA replication forks. Trends Cell Biol 17: 271-278.

Labib K, Tercero JA, Diffley JFX. 2000. Uninterrupted MCM2-7 function required for DNA replication fork progression. Science 288: 1643-1647.

Li Z, Santangelo TJ, Cubonova Lu, Reeve JN, Kelman Z. 2010. Affinity purification of an Archaeal DNA replication protein network. MBio 1: e00221-10.

Li Z, Pan M, Santangelo TJ, Chemnitz W, Yuan W, Edwards JL, Hurwitz J, Reeve JN, Kelman Z. 2011. A novel 
DNA nuclease is stimulated by association with the GINS complex. Nucleic Acids Res 39: 6114-6123.

Liew LP, Bell SD. 2011. The interplay of DNA binding, ATP hydrolysis and helicase activities of the archaeal MCM helicase. Biochem J 436: 409-414.

Makarova KS, Wolf YI, Mekhedov SL, Mirkin BG, Koonin EV. 2005. Ancestral paralogs and pseudoparalogs and their role in the emergence of the eukaryotic cell. Nucleic Acids Res 33: 4626-4638.

Marinsek N, Barry ER, Makarova KS, Dionne I, Koonin EV, Bell SD. 2006. GINS, a central nexus in the archaeal DNA replication fork. EMBO Rep 7: 539-545.

Martin A, Baker TA, Sauer RT. 2005. Rebuilt AAA plus motors reveal operating principles for ATP-fuelled machines. Nature 437: 1115-1120.

McGeoch AT, Trakselis MA, Laskey RA, Bell SD. 2005. Organization of the Archaeal MCM complex on DNA and implications for the helicase mechanism. Nat Struct Mol Biol 12: 756-762.

Moreau MJ, McGeoch AT, Lowe AR, Itzhaki LS, Bell SD. 2007. ATPase site architecture and helicase mechanism of an Archaeal MCM. Mol Cell 28: 304-314.

Moyer SE, Lewis PW, Botchan MR. 2006. Isolation of the Cdc45/Mcm2-7/GINS (CMG) complex, a candidate for the eukaryotic DNA replication fork helicase. Proc Natl Acad Sci 103: 10236-10241.

Pape T, Meka H, Chen SX, Vicentini G, van Heel M, Onesti S. 2003. Hexameric ring structure of the full-length archaeal MCM protein complex. EMBO Rep 4: 1079-1083.

Poplawski A, Grabowski B, Long SF, Kelman Z. 2001. The zinc finger domain of the archaeal minichromosome maintenance protein is required for helicase activity. $J$ Biol Chem 276: 49371-49377.

Remus D, Beuron F, Tolun G, Griffith JD, Morris EP, Diffley JFX. 2009. Concerted loading of Mcm2-7 double hexamers around DNA during DNA replication origin licensing. Cell 139: 719-730.
Rothenberg E, Trakselis MA, Bell SD, Ha T. 2007. MCM forked substrate specificity involves dynamic interaction with the 5'-tail. J Biol Chem 282: 34229-34234.

Sakakibara N, Kasiviswanathan R, Melamud E, Han M, Schwarz FP, Kelman Z. 2008. Coupling of DNA binding and helicase activity is mediated by a conserved loop in the MCM protein. Nucleic Acids Res 36: 1309_ 1320.

Sakakibara N, Kelman LM, Kelman Z. 2009. Unwinding the structure and function of the archaeal MCM helicase. Mol Microbiol 72: 286-296.

Sanchez-Pulido L, Ponting CP. 2011. Cdc45: The missing RecJ ortholog in eukaryotes? Bioinformatics 27: 18851888.

Schwacha A, Bell SP. 2001. Interactions between two catalytically distinct MCM subgroups are essential for coordinated ATP hydrolysis and DNA replication. Mol Cell 8: 1093-1104.

Shin J-H, Heo G-Y, Kelman Z. 2009. The Methanothermobacter thermautotrophicus MCM helicase is active as a hexameric ring. J Biol Chem 284: 540-546.

* Siddiqui K, On KF, Diffley JFX. 2013. Regulating DNA replication in eukarya. Cold Spring Harb Perspect Biol doi: 10.1101/cshperspect.a012930.

* Tanaka S, Araki H. 2013. Helicase activation and establishment of replication forks at chromosomal origins of replication. Cold Spring Harb Perspect Biol doi: 10.1101/ cshperspect.a010371.

Yoshimochi T, Fujikane R, Kawanami M, Matsunaga F, Ishino Y. 2008. The GINS complex from Pyrococcus furiosus stimulates the MCM helicase activity. J Biol Chem 283: 1601-1609.

Yu X, VanLoock MS, Poplawski A, Kelman Z, Xiang T, Tye BK, Egelman EH. 2002. The Methanobacterium thermoautotrophicum MCM protein can form heptameric rings. EMBO Rep 3: 792-797. 


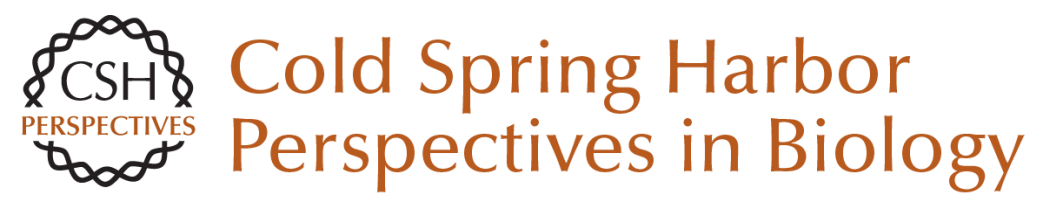

\section{The Minichromosome Maintenance Replicative Helicase}

Stephen D. Bell and Michael R. Botchan

Cold Spring Harb Perspect Biol 2013; doi: 10.1101/cshperspect.a012807 originally published online July 23, 2013

\section{Subject Collection DNA Replication}

Replication of Epstein-Barr Viral DNA Wolfgang Hammerschmidt and Bill Sugden

Replication Proteins and Human Disease Andrew P. Jackson, Ronald A. Laskey and Nicholas Coleman

Break-Induced DNA Replication Ranjith P. Anand, Susan T. Lovett and James E. Haber

Regulating DNA Replication in Eukarya Khalid Siddiqui, Kin Fan On and John F.X. Diffley

Archaeology of Eukaryotic DNA Replication Kira S. Makarova and Eugene V. Koonin

Translesion DNA Polymerases Myron F. Goodman and Roger Woodgate

Human Papillomavirus Infections: Warts or Cancer?

Louise T. Chow and Thomas R. Broker

Chromatin and DNA Replication

David M. MacAlpine and Geneviève Almouzni
Endoreplication

Norman Zielke, Bruce A. Edgar and Melvin L.

DePamphilis

Replication-Fork Dynamics

Karl E. Duderstadt, Rodrigo Reyes-Lamothe, Antoine M. van Oijen, et al.

Helicase Activation and Establishment of

Replication Forks at Chromosomal Origins of

Replication

Seiji Tanaka and Hiroyuki Araki

Poxvirus DNA Replication Bernard Moss

The Minichromosome Maintenance Replicative Helicase

Stephen D. Bell and Michael R. Botchan

DNA Replication Origins

Alan C. Leonard and Marcel Méchali

Principles and Concepts of DNA Replication in

Bacteria, Archaea, and Eukarya

Michael O'Donnell, Lance Langston and Bruce Stillman

DNA Replication Timing

Nicholas Rhind and David M. Gilbert

For additional articles in this collection, see http://cshperspectives.cshlp.org/cgi/collection/

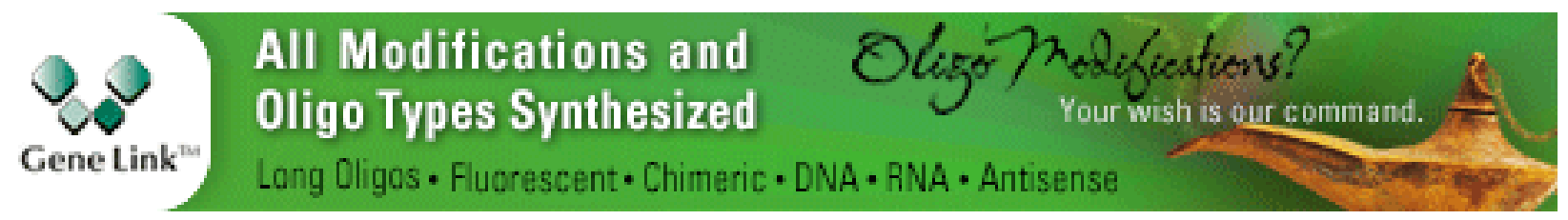

Copyright @ 2013 Cold Spring Harbor Laboratory Press; all rights reserved 